\title{
Modeling activities in air traffic control systems: antecedents and consequences of a mid-air collision
}

\author{
Paulo Victor R. de Carvalho ${ }^{\mathrm{a}, *}$ and Bemildo Ferreira ${ }^{\mathrm{b}}$ \\ ${ }^{a}$ Nuclear Engineering Institute, Cidade Universitária, Rio de Janeiro, RJ, Brazil \\ ${ }^{\mathrm{b}}$ Brazilian Federation of Air Traffic Controllers Association, São Paulo, SP, Brazil
}

\begin{abstract}
In this article we present a model of some functions and activities of the Brazilian Air traffic Control System (ATS) in the period in which occurred a mid-air collision between flight GLO1907, a commercial aircraft Boeing 737-800, and flight N600XL, an executive jet EMBRAER E-145, to investigate key resilience characteristics of the ATM. Modeling in some detail activities during the collision and related them to overall behavior and antecedents that stress the organization uncover some drift into failure mechanisms that erode safety defenses provided by the Air Navigation Service Provider (ANSP), enabling a mid-air collision to be happen.
\end{abstract}

Keywords: air traffic, Brazilian Air traffic Control System (ATS), Air Navigation Service Provider (ANSP)

\section{Introduction}

The air collision between the flight GLO1907, a Boeing 737-800NG, and the flight N600XL, an executive jet Legacy-EMBRAER E-145 on September 29, 2006 over the Amazon rainforest was the biggest Brazilian air traffic accident. The accident has started a cascading series of events that resonated in many areas of the Brazilian society provoking furious searches for "causes and blames". Indeed, after 5 years of investigations 3 people have been condemned both by civil and military justice, but the overall organizational environment wasn't questioned at all regarding its efficiency to prevent this or other huge accident.

The model we present in this article intend to go far beyond the already known "human error" conclusions of the accident investigative teams like lack of skill, miscommunication, inattention, physical and mental workload, poor situation awareness, bad decision making, ineffective action planning, inability to cope with stress, emotional load and so forth [1].

\footnotetext{
* Corresponding author. E-mail: paulov@ien.gov.br
}

Such factors could often be due to a lack of organizational well-being and as pointing out by many authors such "human errors" are consequences not "causes" of accidents [2]. To a better understanding of the accident and on the behalf of the prevention matters the organizational risk management should start from these issues in order to figure out the elements and actions present in the environment to improve safety and resilience.

In this article we model the Brazilian Air Traffic Control of the Brazilian Area Center (ACC-BS) using the Functional Resonance Analysis Method (FRAM) because this tool takes into account the variability inherent in the complex and high-risk systems. FRAM also evaluates how coupled are the subsystems, from loose to tight.

Safety and resilience are emergent organizational properties generated through personal attitudes in an environment grounded on concepts of mindfulness, proactive reasoning, flexibility and adaptability. These issues imply people's cognitive, emotional, motivational behaviors that cannot flourish in rule- 
following or in malaise environments. There it can be developed and disseminated only in open-mind organizations that prioritize taking care of people well-being.

Thus, it is important to change the view that human agents (in the present case either the air traffic controllers or the pilots) are the guilty ones, and the organized actions they organized aimed solely to attract public attention to the ills that they suffered in terms of working conditions, low wages and imputation of guilt in the collision between aircraft. It is also important to overcome the simplicity of the worldwide view spread by the Press as "Air Chaos" or "Air Crisis" representing the basic explanation of what happened in the main Brazilian airports between October 2006 and March 2007.

\section{Method}

The method is based on the analysis of a single case study - the GLO1907/N600XL collision - that can be justified by Yin's (1994) rationale. According to Yin, single case studies must be based in unique and extreme events when how and why questions are being posed, and when the focus is on a contemporary phenomenon within some real-life context.

Mid-air collisions are the least frequent air traffic control (ATC) related accident, and it is revelatory due to the generation of a considerable amount of data that become available to the public and researchers through secondary sources. In our research method, we search for the mid-air collision antecedents traced through the concurrence of performances of the several actors (pilots and controllers) and their interaction with the contextual conditions of the several ATC subsystems. Our aim is to understand how and why this accident happened based on the rich data set that, because the occurrence of this tragedy is now available to the public. Data and evidences, antecedents and consequences from this mid-air collision come from a wide range of publicly available sources. These sources include official government documents, congressional hearings, including controllers', pilots', and air traffic management authorities' testimonies, video tapes, audio tapes of many media centers, press releases, newspaper clippings, flight plans, regulations, maps, directives and so forth.

Based on this data We use the Functional Resonance Accident Method - FRAM [3] to model the functions and activities of the ATM system. The basic steps to perform an analysis using FRAM are: 1) define the purpose of modeling (risk assessment) and describe the target situation or scenario to be analyzed; 2) identify essential system functions, characterize each function by six basic parameters (Input, Output, Time, Control, Precondition, Resource); 3) characterize the context-dependent observed and potential variability of system functions; 4) identify and describe functional resonance from observed dependencies/couplings among the functions and observed variability; 5) identify control mechanisms or barriers for variability (damping factors) and specify required performance monitoring.

\section{Brazilian air space control system}

The Brazilian Airspace Control System (SISCEAB) is a set organizations composed by highly skilled workers and technology to manage the flow and safety of civilian or military (in peacetime operations) aircraft when using Brazilian airspace. The Department of Airspace Control (DECEA) is the central body of and accumulates for itself other three systems: SPV- Flight Protection System; STCA - Air Force Telecommunications System; and SISSAR Search and Rescue System. To manage and operate these systems, DECEA incorporates high specialized activities and has twelve subordinate units, also called DECEA's military organizations that act directly on the deployment and maintenance of systems and resources and on the implementation of operational activities. They are the implementing bodies that execute the goals established by the central body. DECEA are distributed into three Sub departments of supervision, and regulate four Integrated Centers of Air Defense and Air Traffic Control (CINDACTA), one Regional Service of Flight Protection established in São Paulo (SRPV-SP), five Area Control Centers (ACC), 47 Approach Controls (APP), 59 Air Traffic Control Towers (TWR), 79 Regional Air Space Control Section (DTCEA), in addition to more than 90 Aeronautical Telecommunications Stations and various support divisions across the country. In addition to continuous training and development of the institution's human resources for the implementation, planning and management of the systems, the deployment of infrastructure and maintenance of its operational centers, DECEA operates directly implementing activities of the three systems SPV, STCA and SISSAR 


\subsection{The Air Traffic Control Service (ATS)}

According to ICAO regulations, Air Traffic Control Services (ATS) units are provided by air traffic control in order to prevent collisions between aircraft and other aircraft with possible obstructions in the maneuvering area, and to issue and maintain its flow. They are units of air traffic control, the generic bodies operating control tower (TWR), Approach Control (APP) and Area Control Center (ACC). Each has its particular geographical jurisdiction in service delivery. However, there are situations that they can be merged or are delegated as a facility that provides the services of another. Hierarchically, the sequence is ACC, APP and TWR and the services provided meet the aircraft on the route, aircraft descent into landing, or ascent to the route, aircraft about eight kilometers from the airport, and aircraft moving in the airport runways, respectively.

\subsection{The air traffic controller (ATC)}

The core activity of the air traffic controller is to separate aircraft that fly in their jurisdiction using the minimum distance or time stipulated by the Brazilian aviation authority. In Brazil there are civilian and military controllers working in the same system. Most military controllers are sergeants in the aeronautic graduate at the Air Force School of Specialists (EEAR). Civilians, in turn, are divided into four groups, all of them trained by ICEA: civilians who work directly for the Brazilian Air Force (FAB) who public servants called "DACTAS" - Air Defense and Air Traffic Control; civilian hired by agreements with international institutions such as ICAO or nonprofit organizations advising the aviation authority, calls OSCIP - Civil Society Organization of Public Interest, civilians employed by private companies that have granted by the authority to operate in the aeronautical industry, and finally, civilians working for the organization that manages the main Brazilian airports (Infraero). The most visible difference between the capabilities of the controllers trained by EEAR and ICEA is the military training.

\subsection{Automation in the Area Control Centers (ACC)}

The increased use of airspace, the enhanced flight performance and embedded aircraft result in a need of the automation of controller tasks to support decision making. As already discussed by Fulton (2010) the air traffic control technology will have an expo- nential grow in the next years. In Brazil, the dimensions of the various airspace control sectors remained almost the same since they were created in the mid1970s. In some facilities, the air traffic controllers separate the traffic based on radar information that have evolved from a simple presentation of a primary target (a green spot in a black screen) to display many target information in a 21 " screen or even larger displays called "video-maps." This display contains a wealth of information about the traffic and the area of jurisdiction as well as the electronic flight strips showing the mnemonic representation of the instructions given to the aircrafts by the controllers in the various sectors. At January 25, 2005, an important change occurred with the implementation of the Reduced Vertical Separation Minima (RVSM) (DECEA, 2004), meaning that more aircraft could be allocated in the same airspace. This is done according to the new industry regulations, because the accuracy and reliability of new-generation avionics and technical-operational counterparts on land should be enough to safely prevent the loss of vertical separation.

\section{The midair collision}

The collision occurred at 1656 Brazilian time (1956 UTC) on September 29, 2006 about 1 hour after the EMB-135BJ Legacy airplane, flight N600XL, entered the air-space controlled by the Brasilia ACC. Flight N600XL collided with flight GLO1907, a the Boeing 737-800, in the surveillance intersection zone between Amazon ACC (AZ ACC) and Brasilia ACC (ACC BS). The collision cut the wing of the Boeing, causing the pilots to lose control, and flight 1907 immediately dove into the ground, causing the death of all passengers and crew members (154 people). The N600XL, with some damage, was able to land at a military base airport.

Flight N600XL took off from São Jose dos Campos (SJC) airport at 1430 (Brasilia time) bound for Eduardo Gomes airport in Manaus (Amazon) with two crew (pilot and co-pilot) and five passengers. It was cleared for flight level 370 ("November six zero zero x-ray lima, ATC clearance to Eduardo Gomes, flight level three seven zero..."). However, the N600XL flight plan had three different cruising altitudes: level 370 (37,000 feet) along the UW2 airway, crossing the sector 5, up to the Brasilia vertical line (BRS VOR), level 360, when the aircraft entered in sector 7 flying along the UZ6 airway up to 
TERES position, and level 380 from TERES to Manaus. Therefore, the route planned for the N600XL passed through the sectors 5 and 7 of the ACC BS.

Flight GLO1907 took off from Eduardo Gomes airport in Manaus bound for Brasilia International Airport, cleared for flight level 370 by ATC of AZ ACC, maintaining this level up to the collision.

The first flight level change of the N600XL to FL360 should occur just before the BRS VOR, still inside the sector 5 of ACC BS. At 155037, around 5 minutes before the N600XL leave sector 5, the sector 5 controller called the N600XL and commanded the switch of the communication frequency to 125.05 $\mathrm{MHz}$. There was no mention about the need to change flight level in a near future. The hand off of N600XL from sector 5 ATC to sector 7 ATC was made just after the reply of N600XL crew, when the aircraft was still inside sector 5 (about five minutes before BRS VOR). As a consequence, the sector 7 ATC received the traffic outside his control area, on the frequency of $125.05 \mathrm{MHz}$ which, according to the frequencies chart, was not the most adequate frequency to be used in sector 7(should be. $135.90 \mathrm{MHz}$ ).

The last bilateral contact between flight N600XL and the Brasilia ACC, at 155104, occurred with the sector 7 ATC, while the N600XL was still in sector 5, but controlled by sector 7 , who has just received the anticipated traffic. The controller who assumed the control was the one responsible for sectors 7, 8 and 9, and operating at console 8 . In this last bilateral communication, the sector 7 controller, using the frequency $125.05 \mathrm{MHz}$, received the information from the N600XL co-pilot that he was flying at FL370 (correct for sector 5), and in reply confirming that the N600XL was under radar surveillance (RVSM air space), again there is no mention to flight level changes in near future.

At 155548, the N600XL flew over the Brasília VOR vertical line (entering in sector 7), reaching the UZ6 airway keeping the FL370 (the same level as the one cleared to flight 1907, in the opposite direction). Flying under positive control and RVSM conditions, the pilots were not obligated to report when passed over the vertical of BRS VOR and, according to their understanding of the initial clearance, they keep FL370, as indicated in Figure 1.

The left field of the data block receives accurate altitude information while the aircraft transponder is operating in Mode C (when the transponder is transmitting aircraft altitude information) and indicated the current flight level (370). The right field of the data block changes automatically the data displayed about two minutes before the point where the expected level change is supposed to occur. The right field changed from the cleared or authorized flight level (370 sector 5 flight level) to the requested flight level for the next route segment (sector 7 flight level, 360). This automatic change is made according to the aircraft active flight plan and the system does not warn the controller in any special way.

Fig. 1 N600XL data block over BRS VOR.

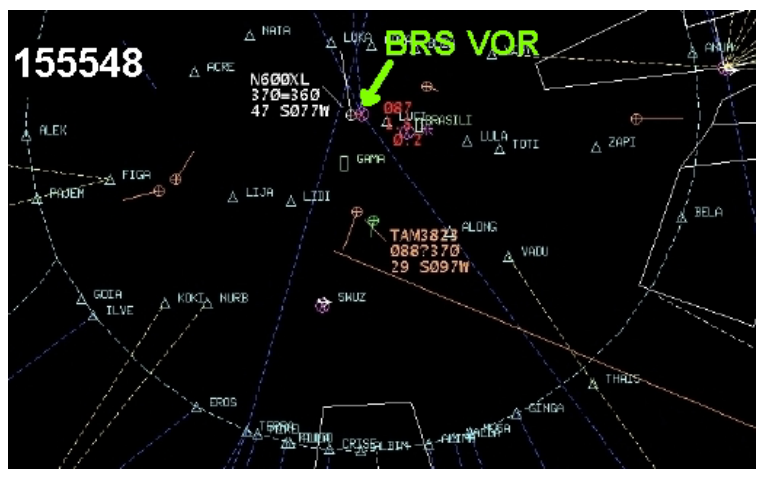

In Figure 1, the N600XL data block indicates $370=360$ when the aircraft flew over BRS VOR. It means that FL370 is the current altitude, informed by the aircraft transponder operating in Mode $\mathrm{C}$, the = signal indicates aircraft stabilized (nor climbing or descending), and FL 360 is the level requested for next segment of the route that started at BRS VOR. The indication $370=360$, meaning that there was an aircraft about to fly or flying at a flight level that was different from the flight level requested in the active flight plan, remained for seven minutes in the display, and during this period there were no calls to or from the N600XL. The "=" signal means that the aircraft was leveled. The left field changed from the cleared or authorized flight level, i.e., from the FL370 in the previous sector to the requested flight level - in the active flight plan - to FL360 in the next route segment, i.e., in sector 7. This change is done by the radar console automatically and it is attached to the aircraft active flight plan. The active flight plan can be different from the current flight plan when the necessary ATC instructions is applied to the aircrafts for the sake of better and safe separation from other aircrafts. The software in this particularly radar console does not provide any aural or visual warnings to the air traffic controller when the automatic changes 
occur. Seven minutes later, at 1602, the N600XL transponder stopped the transmission to the secondary surveillance radar (SSR) of the ACC BS, interrupting the mode $\mathrm{C}$ accurate altitude information to the controller. In this situation, the altitude information comes from the primary (3D or height finding) radar and it is not as accurate as the altitude information obtained from transponder signals. In this situation, the data block automatically changed from $370=360$ to $370 \mathrm{Z360}$ ( $\mathrm{Z}$ indicated that the altitude information come from $3 \mathrm{D}$ radar). The loss of Mode $\mathrm{C}$ information also changes the shape of aircraft position icon; the aircraft is represented only by a cross associated with the vector line, without the circle. Again, the system does not warn the controller about the change. At this moment the N600XL data block displayed various inaccurate altitude information (the 3D radar generated altitude measurement), which clearly should not be used for the provision of vertical separation between aircraft. After the loss of transponder signals there were no calls to or from the N600XL.

At 1915 there was a shift changeover in the ACC $\mathrm{BS}$ sector 7. According to the final accident report [4], the controller being relieved and the relieving ATC did not mention any abnormalities affecting the N600XL.

At 1624, the controller manually changed the CFL field (cleared flight level) of the N600XL electronic flight strip of the next route segment to FL360. Contrary of the automatic changes that occurred in the data block displayed in screen, the CFL field in the electronic strip requires the controller to insert the flight level he authorized for each route segment. The change made by the controller of ACC BS to FL360 caused the change in all subsequent strips, including the one at the ACC AZ (to where N600XL was going), to FL360, as if the aircraft had been authorized by the controller and was actually flying at FL360. The action indicates that the sector 7 controller, at 1924, was still convinced that the N600XL was maintaining flight level FL360.

Neither flight N600XL, nor the Brasilia ACC tried to contact each other between 1551 and 1626. After 1626, the ACC BS made 7 unsuccessful call attempts, using six frequencies that were previously selected on the control console number 08 (135.9/ 125.2/ 125.05/ 133.1/ 122.25 and $125.45 \mathrm{MHz}$ ). Despite being transmitting on $125.05 \mathrm{MHz}$ (the one commanded for N600XL), the aircraft was beyond the range of this frequency. At 162712, about 30 minutes after the N600XL entered in sector 7, a frequency change to $135.9 \mathrm{MHz}$, (the sector 7 north frequency) was commanded. However, there was not reply from N600XL to the call. The same happened with the other call attempts. At 1638, ACC BS lost definitively the $3 \mathrm{D}$ radar contact with flight N600XL (it should be transferred to the AZ ACC).

\section{Modeling activities using FRAM}

The basic step to perform an analysis using FRAM [3] is the identification essential system functions and the characterization of each function by six basic parameters (Input, Output, Time, Control, Precondition, Resource), in order to understand the potential variability in system functions to identify functional resonance possibilities. The final aim is to develop control mechanisms or barriers to avoid or damp resonances.

Based on the accident description we use FRAM to model the flight control functions that include cockpit flight monitoring, the Traffic Alert and Collision Avoidance System - TCAS function, the air traffic monitoring function, and the communication function, as shown in figure 2. The FRAM diagram indicates that the flight monitoring and control are based on a set of human centered loosely coupled functions, which due to its own nature, are subject to possible unwanted (but normal due ETTO principle [3]) variability, especially if some pre-conditions and basic resources for the proper operations of these functions are not fulfilled. As displayed in fig.2, the combination or resonance among functions variabilities lead to the disruption of control loops (dotted lines), and the entire set of functions responsible to flight control in RVSM space were not able to prevent the mid-air collision.

The early handoff from sector 5 to sector 7 controller, together with the automatic change in the CFL field contributes to trigger work related ETTO rules such as "it will checked later by someone else / it has been checked earlier by someone else" and the control loop remain open (see fig. 2). The combination of these two rules is clearly unhealthy, because it opens the control loop at the input, when both controllers believe that there is no need to communicate new flight levels to the aircraft (the action was already done by other controller).

At 1602 the controller screen changed automatically because the N600XL transponder signal failed. In case of transponder failure, the controller has to 
suspend RVSM operations. However, even considering the importance that the loss of the transponder signal has in the regulations and in the controller actions, there is no active warning signal delivered by the software system. To perceive the situation, the controller must actively seek for the information on the screen that enhance the possibilities for an unwanted output of the function (transponder failure not perceived) especially because there is a time window involved. In this event, the controller on duty did not take any action, and no communication occurred. After the shift changeover (1615), the new controller waited for 10 minutes before trying the contact with the N600XL, while the aircraft was leaving the Brasilia ACC air space and the signals coming from the $3 \mathrm{D}$ radar system were erratic.

The communication function is the way to close the loop to control controller-pilot system. From 1551 to 1626 there was no communication, despite the transponder problems and sector change. However, if the controller did not perceive changes in the radar screen, or did not perceive such changes as important enough to send instructions, he also did not communicate to the pilots.

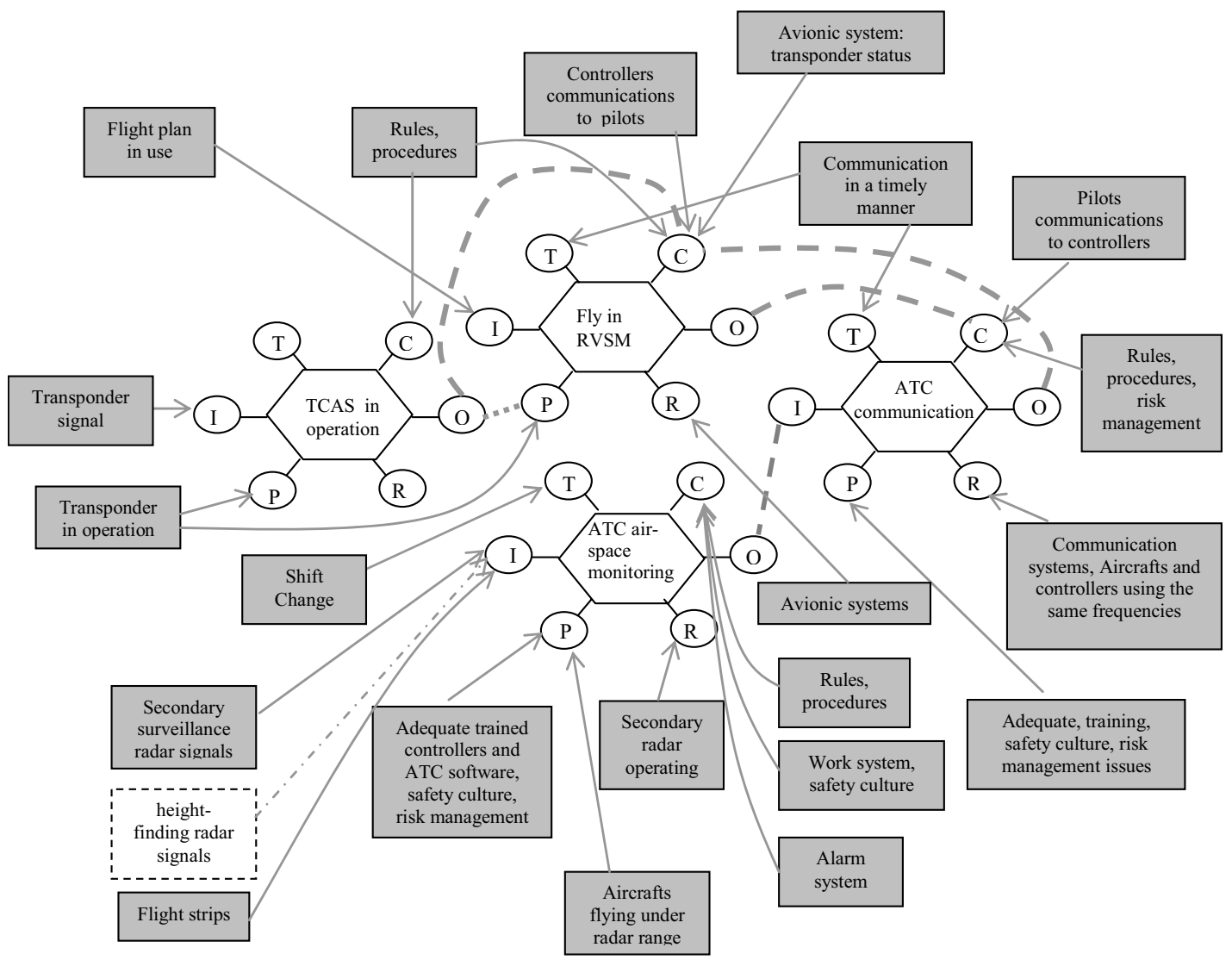

Fig. 2 - Modeling flight monitoring and control functions

\section{Discussion}

If it is not possible in a complex system to constrain performance variability into a set of previously fixed rules, more important becomes the evaluation of its basic resilience characteristics in system operation such as: buffering capacity, flexibility, margin, and tolerance.

Buffering capacity relates to the size and/or kind of disruptions the system can absorb or adapt to without a fundamental breakdown in performance. FRAM indicated that the accident happened when an 
aircraft was flying in RVSM space without adequate control. The basic system design, similar to other ATM systems around the world, had many possibilities to restore control, closing the feedback loops of monitoring functions. However, despite the recovery possibilities, function variabilities resonate in way that lead the system to operate with a low buffer capacity. The pilots were out of the control loop since the beginning of flight, waiting for controllers' instructions, and controllers, using the information available, were not able to perceive the conflict situation in any moment, showing a system operating with low buffering capacity. Situations that contribute to lower buffer capacity of system functions are the unavailability of the frequencies of neighbors sectors in the controllers console (lower buffering capacity of communication function), the lack of alarms about transponder situation and visualization constraints in the controllers' radar scope, the inexistence of Short Term Conflict Alert (STCA) and Separation Monitoring Function (SMF) that adds independent alerting logic to the control loop by generating indications of existing or pending situations, related to the proximity of aircraft as well as their relative positions and speed, which require attention/action (diminish the buffering capacity to monitor many flights together).

Flexibility relates to the system's ability to restructure itself in response to external changes or pressures. Flexibility emerge as new couplings between functions that were not planned and instantiated before, possibly in combination with the introduction and performance of new functions during system functioning. Thus, flexibility and "new" behavior can be identified through new couplings and functions in the model that enhances system performance. Based on this view the ATS showed very little flexibility. This may be exemplified by the aircraft flying without control in a RVSM air space, in a wrong air way, without being perceived by any other ACC. When the main control loop, i.e., the controller in the ACCBS and the aircraft N600XL, did not work, any other control function appeared to restore the control loop to perceive the dangerous situation.

Margin relates to how closely or how precarious the system is currently operating relative to a certain performance boundary. It seems in this mid-air collision that the margin was changing over time by unperceived performance variations that lead to a normal performance, for example, in clearance communications, that was different from the performance described in the rules and procedures, or in the need to communicated with and aircraft that enters in the controller sector, when this aircraft did not commu- nicate previously, where there is no clear instructions. These performance variations were developed over time and, even considering that there are no absolute boundaries of safe performances, it showed a trend in margin decline. A more realistic view of margins and their development is obtained when various instantiations of the model (splitting the functions described here in other, lower level, functions), and their couplings over time (especially in loosely coupled situations), are compared. Therefore the actual (normal) performance compared to various margins can be analyzed using the Cognitive Task Analysis (CTA) methods together with a combination of function controls, in order to show how actual performance is chosen to met the various operational/personal objectives (e.g., ETTO behavior), and to cope with system constraints. The CTA method together with instantiations of a FRAM model in different ATS system functions may help address these issues in a structured way, by following and assessing potential and actual couplings between functions in a system over time shedding some light in questions like: Which functions, and people, trade-offs, and processes lay as a basis for the performance boundary setting? How do these functions (trade-offs, processes) drift over time, and how does the boundary change over time influenced by these same functions? How does the performance of these functions vary, and how are they interconnected?

Tolerance relates to how a system behaves near a boundary, for example along the lines of graceful degradation or quick collapse, when pressure exceeds adaptive capacity. The design of the ATS work systems (work organization, rules procedures, and instructions) and subsequent supervision and coordination aim at foreseeing to avoid performance adjustments that may lead to unwanted outcomes in system performance. This design characteristic is especially important in systems that do not have physical safety barriers like the airways to the ATS. FRAM analysis has shown that the lack of control that lead in the collapse of overall system performance occurred when various (small) performance adjustments resonate to produce a much bigger outcome, which may indicate that the system has a low tolerance for some performance variations. Therefore, the system tolerance can only be properly understood when performance adjustments are analyzed combined with other controls and work conditions. Given the possibility of a quick collapse among normal performance variations, this accident shows the need of continuous performance monitoring of system functions, in order 
to understand the rationale behind the adjustments, and to damp unwanted variations in human performance.

Cross-scale interactions relate to how a system defined at one scale depends on influences from systems defined at other scales [9]. Therefore the assessment of interactions between various systems is highly dependent on the definition of system boundaries. In FRAM, influences among systems appear as couplings among functions. As an example, if the pilots were not aware of the flight plans and local air charts, they become out of the control loop, and the system misses cross scale interaction opportunities. Therefore the position/responsibilities of pilots in the ATS system limits cross scale opportunities within the system. Cross check opportunities to close the control loop were missed when the N600XL changed sectors (from sector 5 to sector 7) and during the shift changeover.

\section{Conclusion}

It has for a long time been taken for granted that accidents must explained by failures and malfunctions. In this collision, all equipment functioning according their project specifications (even the TCAS). Using this rationale, if equipment did not fail then people must be failed. Human error appears when people try to create safety and better operating through their performance adjustments to deal with multiple (conflicting) goals in complex (dynamic) systems. Our analysis of the system operation through the lens of the collision, or based on normal situations in which the collision emerged showed that people behavior sometimes were different from those prescribed in rules and procedures. The traditional safety approach may conclude that the human errors such as lack of adherence to rules and procedures, lack of attention during monitoring appeared as cause of the accident, and basic recommendations were directed to improve people behavior or to get better people to work.

FRAM modeling indicated that the flight monitoring and control were based on a set of human centered loosely coupled functions which, due to its own nature, were subject to possible unwanted variability although considered normal due the ETTO principle (Hollnagel, 2009), especially if some pre-conditions and basic resources for the proper operations of these functions are not fulfilled. The combination or resonance among functions variabilities led to the disruption of control loops and the entire set of functions responsible to flight control in the RVSM space were not able to prevent the mid-air collision. Our analysis indicated also that performance adjustments or variability could be explained by ETTO principle, and sometimes performance adjustments were nearly required and they seemed to be the way in which people did their work to cope with environment constraints. Although performance variability is both necessary and useful, there will also be situations where it leads to unwanted and unintended outcomes, especially in a tight-coupled and timely constraint system such as the ATS, where consequences may develop rapidly and potentially be very serious with no know or available paths of recovery. The early handoff from sector 5 to sector 7 controller, together with the automatic change in the Active Flight Plan -distinct the from the Current Flight Plan already modified by the air traffic controller -- field contributes to trigger work related ETTO rules such as "it will checked later by someone else / it has been checked earlier by someone else" and the control loop remain open (see fig. 2). The combination of these two rules is clearly unhealthy, because it opens the control loop at the input, when both controllers believe that there is no need to communicate new flight levels to the aircraft (the action was already done by other controller).

\section{References}

[1] Senado Federal. Relatório dos trabalhos da CPI do apagão aéreo. Brasília: Senado Federal; 2007 (in Portuguese).

[2] Dekker S. Ten Questions About Human Error. London: Laurence Erlbaum; 2005.

[3] Hollnagel E. Barriers and Accident Prevention. Aldershot, UK: Ashgate; 2004.

[4] Centro de investigação e prevenção de acidentes aeronáuticos - CENIPA. Relatório Final A-022/CENIPA. Brasilia: Estado Maior da Aeronáutica; 2008 (in portuguese). 